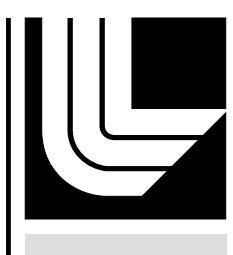

LAW RENCE LIVERMORE N A TIO NAL LABORATORY

\title{
Localized functionalization of single nanopores
}

J. Nilsson, J. R. I. Lee, T. V. Ratto, S. E. Letant

September 13, 2005

Advanced Materials 
This document was prepared as an account of work sponsored by an agency of the United States Government. Neither the United States Government nor the University of California nor any of their employees, makes any warranty, express or implied, or assumes any legal liability or responsibility for the accuracy, completeness, or usefulness of any information, apparatus, product, or process disclosed, or represents that its use would not infringe privately owned rights. Reference herein to any specific commercial product, process, or service by trade name, trademark, manufacturer, or otherwise, does not necessarily constitute or imply its endorsement, recommendation, or favoring by the United States Government or the University of California. The views and opinions of authors expressed herein do not necessarily state or reflect those of the United States Government or the University of California, and shall not be used for advertising or product endorsement purposes. 


\title{
Localized functionalization of single nanopores
}

\author{
Joakim Nilsson, Jonathan R. I. Lee, Timothy V. Ratto, and Sonia E. Létant ${ }^{*}$
}

Chemistry and Materials Science Directorate, Lawrence Livermore National Laboratory

7000 East Avenue, Livermore, CA 94550, USA

* Corresponding author. Mail code: L-232, E-mail: letant1@1lnl.gov, Phone: 925-423-9885.

\begin{abstract}
We demonstrate the localization of chemical functionality at the entrance of single nanopores for the first time by using the controlled growth of an oxide ring. Nanopores were fabricated by Focused Ion Beam machining on silicon platforms, locally derivatized by ion beam assisted oxide deposition, and further functionalized with DNA probes via silane chemistry. Ionic current recorded through single nanopores at various stages of the fabrication process demonstrated that the apertures can be locally functionalized with DNA probes. Future applications for this functional platform include the selective detection of biological organisms and molecules by ionic current blockade measurements.
\end{abstract}


The Coulter-counter principle used for cell enumeration in hematology laboratories is based on the fact that the ionic current through a single aperture drops each time a cell obstructs the channel by moving through it. ${ }^{1-2}$ Although much work has been devoted to the reduction of aperture diameters to count smaller and smaller biological objects, ${ }^{3-10}$ the next challenge remains to combine size control with local chemical functionality to achieve specific counting and detection. The initial solution to this problem was provided by the use of $\alpha$-hemolysin, a cellular membrane protein that self-assembles in lipid bilayers to form a $1.5 \mathrm{~nm}$ diameter pore. Bayley et al. ${ }^{11-12}$ showed that a single DNA probe could be immobilized at the entrance of the protein pore via genetic engineering and that specific binding events to complementary DNA strands could be detected. The drawback of this approach is that the fluidity of the bilayer platform generates instabilities, e.g. the protein can diffuse to the edge of the lipid membrane and denature, and therefore leads to extremely short functional lifetimes of the pore. Additionally, there is no possibility to tune the pore length and diameter in order to accommodate long DNA strands or larger biological objects such as viruses or bacteria. An alternative approach is to build apertures on a sturdy solid platform. Golovchenko et al. ${ }^{13}$ achieved pore diameters down to $1.8 \mathrm{~nm}$ by ion beam sculpting on a pre-patterned $\mathrm{Si}_{3} \mathrm{~N}_{4}$ membrane but the main issue with this design is that no effective surface chemistry is actually available to provide chemical functionality to the aperture. In an attempt to combine size control with chemical selectivity, Martin et al. ${ }^{14}$ recently isolated a conically shaped gold nanotube and covalently attached single-stranded DNA molecules on the entire membrane via a thiol-terminated linker. The main drawbacks associated with this technique are that the fabrication process is tedious, and that no localization of the chemical linkers is possible as the entire device, including pore wall and surface, is covered with gold. The approach that we will present in this article is to fabricate single pores on pre-patterned silicon platforms by Focused Ion Beam (FIB) drilling, and to locally derivatize the pore entrance via the controlled growth of an oxide ring followed by specific functionalization of the oxide with silane chemistry. Focused Ion Beam nano-machining provides size tunability from microns to nanometers, which will allow biological objects ranging in size from cells 
down to viruses and DNA to be detected. In addition, the combination of ion-assisted deposition and basic silane chemistry provides local chemical functionality at the entrance of the nanopore, which will allow non-fouling coatings to be applied to the surface of the device for optimal stability and improved detection limits.

Silicon on Insulator (SOI) wafers with a device layer thickness of $700 \mathrm{~nm}$ and a handle thickness of $300 \mu \mathrm{m}$ were coated with silicon nitride, patterned, and etched in order to obtain individual $100 \times 100$ $\mu \mathrm{m}$ silicon membranes (see Figure 1). Temporary chromium locator lines were fabricated on the top of the device in order to locate the membrane area in the FIB. These lines were dissolved in a selective chromium etch bath after drilling (CR-7S from Cyantek Corporation).

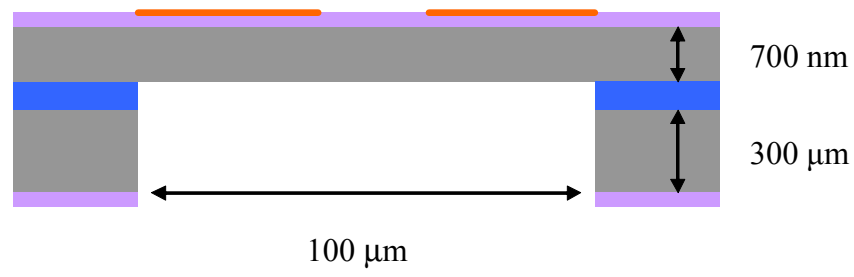

Figure 1. Cross section of a pre-patterned silicon device showing the $100 \times 100 \mu \mathrm{m}$ silicon membrane built on an SOI wafer. Chromium locator lines (orange) are fabricated on the top of the nitride layer (purple) to locate the membrane area in the FIB.

We used a FIB combining electron and ion beam capabilities (Strata dual beam from FEI) for both imaging and fabrication of the devices. ${ }^{15-16} \mathrm{The} \mathrm{Ga}^{+}$ion beam allows ion beam-assisted drilling and deposition while the electron beam allows in situ characterization such as scanning electron microscopy (SEM) and Energy Dispersive x-ray Spectroscopy (EDXS). Varying the $\mathrm{Ga}^{+}$ion beam current from 10 to $50 \mathrm{pA}$ and adjusting the drilling time from less than $10 \mathrm{~s}$ to several minutes on a $700 \mathrm{~nm}$ thick membrane lead to pore diameters ranging from tens of nanometers up to several microns. This demonstrates the versatility of the technique, which offers the potential to adjust the aperture diameter for biological objects ranging in size from DNA to bacteria. The pores used for functionalization experiments had initial diameters ranging from $1000 \mathrm{~nm}$ to $500 \mathrm{~nm}$ prior to oxide deposition. 
After the initial pore drilling, Tetraethylorthosilicate (TEOS) gas was let into the chamber and the ion beam was focused on the area where deposition should take place with a current of $10 \mathrm{pA}$. The pressure in the sample chamber was below 1e-6 mbar while drilling and about $2.5 \mathrm{e}-5$ mbar during oxide deposition. The pores were imaged with the scanning electron beam immediately after fabrication. Figure 2 shows how various pore diameters and oxide thicknesses can be achieved, according to the fabrication conditions. The height of the oxide ring can be controlled by the deposition time, and depends on the diameter of the ring and the current of the ion beam.

(a)

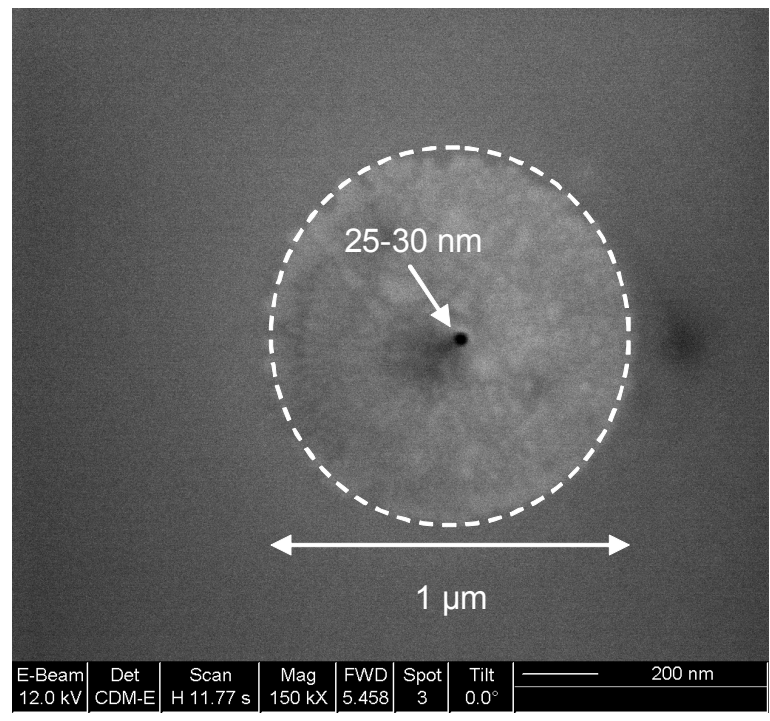

(b)

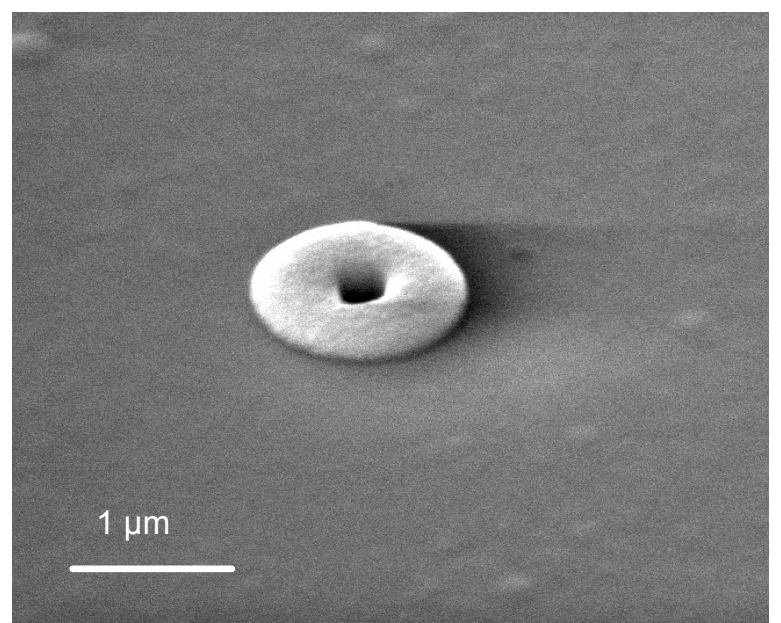

(c)

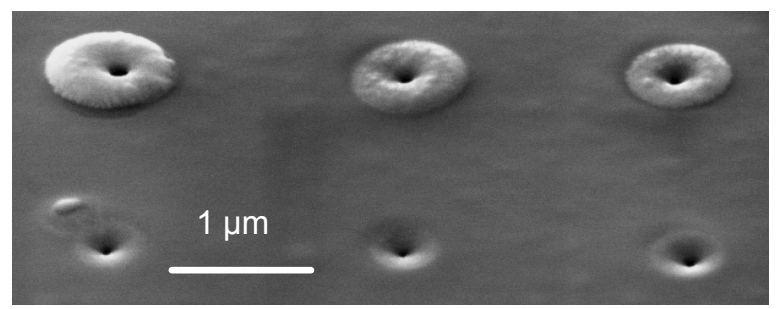


Figure 2. FIB-fabricated pores after local oxide deposition. (a) A pore that has been shrunk from a starting diameter of $1 \mu \mathrm{m}$ down to $25-30 \mathrm{~nm}$. The pore was confirmed to be open with IVmeasurements in $1 \mathrm{M} \mathrm{KCl}$. (b) SEM image of a larger pore $(250 \mathrm{~nm})$ and (c) an array of pores with varying diameters and oxide thicknesses. The pores on the top row were exposed to oxide deposition for several minutes and the pores on the bottom row were exposed for just tens of seconds.

A more detailed topography of the ion beam-assisted TEOS oxide is provided in the AFM picture presented in Figure 3. Pores were imaged in contact mode with a Digital Instruments NanoScope IIIa (Veeco, Santa Barbara, CA) controlling a Bioscope Atomic Force Microscope (AFM). Standard $\mathrm{Si}_{3} \mathrm{~N}_{4}$ DNP-s (Veeco, Santa Barbara, CA) cantilevers with nominal spring constants of $\sim 0.1 \mathrm{~N} / \mathrm{m}$ were used for all scans. The combination of SEM and AFM pictures illustrates how the oxide is indeed localized around the aperture.

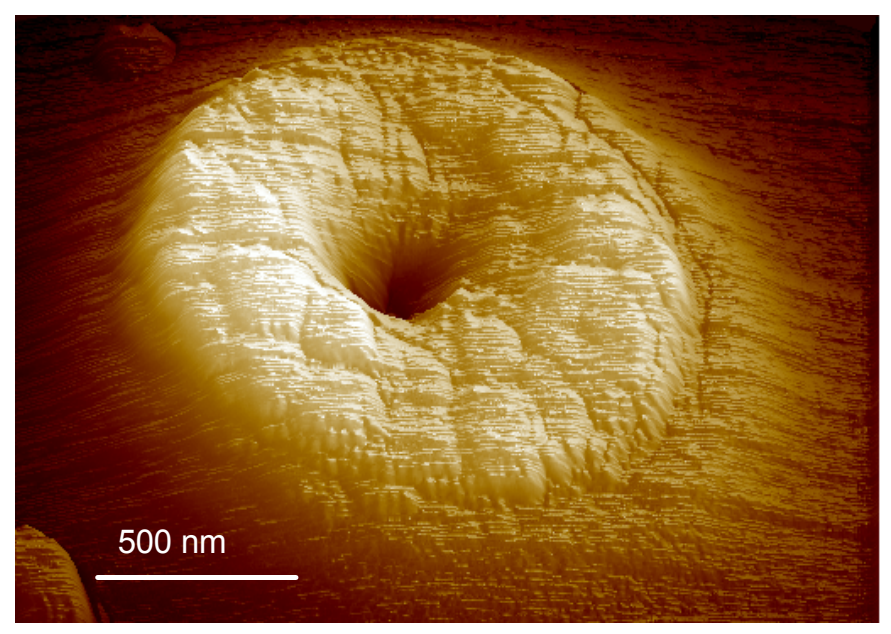

Figure 3. AFM image of a pore after local oxide deposition. The horizontal grooves seen in the image are artifacts of the scan due to vibrational noise and not actual features. The pore diameter is 160 $\mathrm{nm}$ in diameter and the oxide height is $80 \mathrm{~nm}$. 
Maps of the chemical composition of the TEOS oxide ring with 1-2 atomic percent accuracy were obtained using energy dispersive x-ray spectroscopy (EDXS), Figure 4 shows the oxygen map recorded on a ring grown to have a height of about $100 \mathrm{~nm}$, as well as the individual spectra, and composition in atomic percents. For comparison, Figure 4 includes data from a $250 \mathrm{~nm}$ thick oxide ring, illustrating that the oxide content increases and suggesting that the oxide content can be controlled by the growth time. The Ga contamination is due to the ion beam used for both drilling and deposition. The carbon contamination is hard to completely suppress but can be decreased by avoiding direct imaging of the oxide ring with the electron beam.

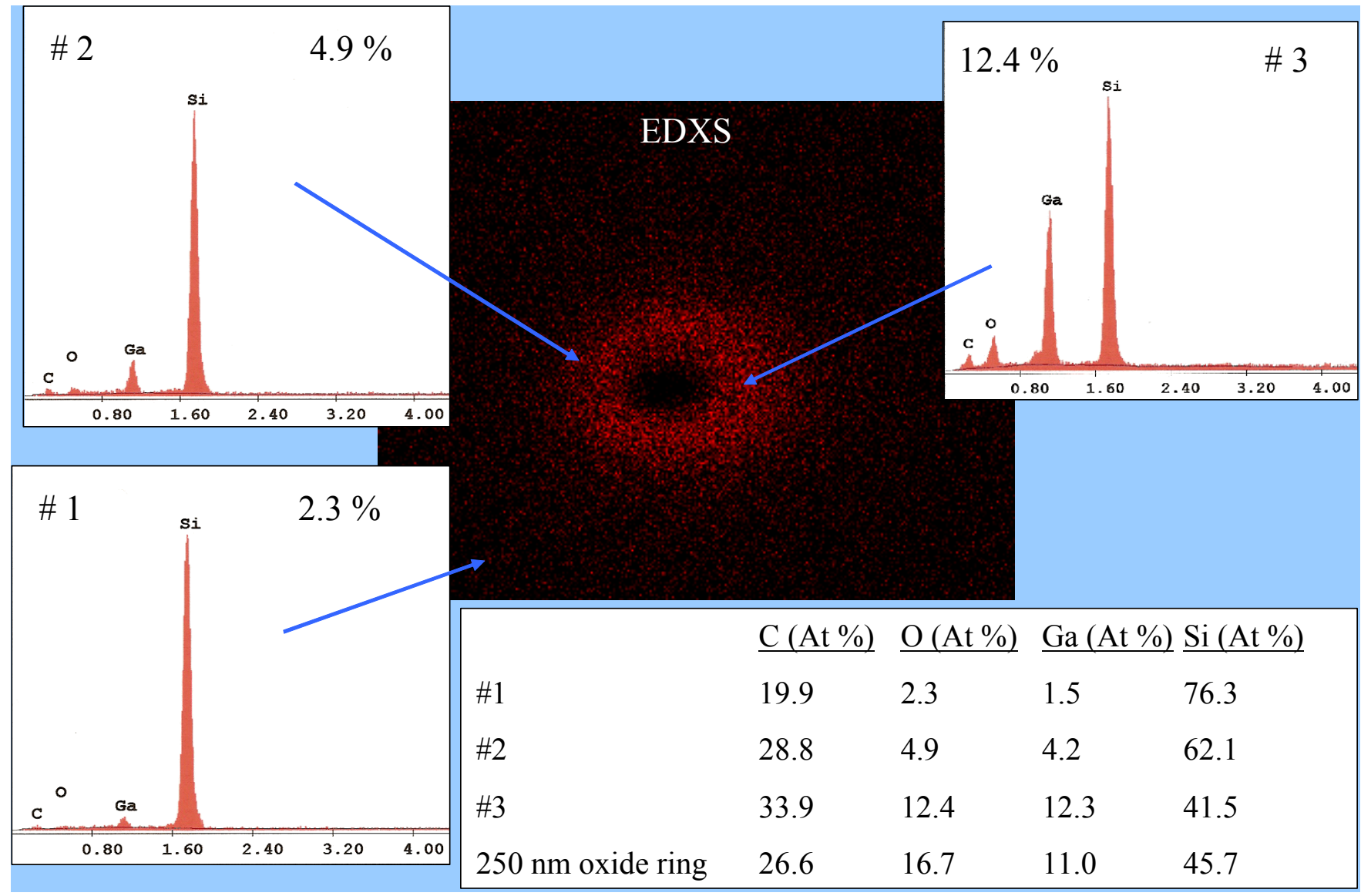

Figure 4. Oxygen map and spectra of a TEOS oxide ring grown by ion beam-assisted deposition in a FIB around a single pore. The chemical composition in atomic percent is shown for various locations around the pore, as well as for a thicker oxide ring. 
Silicon oxide rings were selectively derivatized with alkanethiol linkers via silane chemistry and further functionalized with acrylamide-terminated single stranded DNA probes (ssDNA). Samples were loaded into a glove box under a nitrogen atmosphere with a relative humidity around $15-20 \%$. Inside the glove box the samples were immersed in a solution of $0.1 \mathrm{~mL}$ 3-mercaptopropyltrimethoxysilane (MPTS, from Gelest), and $10 \mathrm{~mL}$ toluene (anhydrous, $99.8 \%$ from Sigma-Aldrich) for 4 hours. They were then rinsed in three $10 \mathrm{~mL}$ aliquots of toluene followed by two $10 \mathrm{~mL}$ aliquots of dimethylformamide (DMF), (anhydrous $99.8 \%$, from Sigma-Aldrich). After each rinse the samples were dried with nitrogen. Dry single stranded acrylamide terminated-DNA (sequence 5'-ATC CTT ATC AAT AAT-3', from IDT) was re-suspended in $150 \mathrm{mM}$ phosphate buffered saline (PBS, from SigmaAldrich). A solution of $1 \mu \mathrm{M}$ was freshly prepared in DMF and transferred into the glove box. The rinsed and dried samples were immersed in the DNA solution and moved to the load lock, where they reacted for 2 hours. After this step, the samples were moved to a fume hood and rinsed with $10 \mathrm{~mL}$ DMF, $10 \mathrm{~mL}$ PBS, and $10 \mathrm{~mL}$ deionized water.

For reference purposes, PECVD TEOS was deposited on $\sim 1 \mathrm{~cm}^{2}$ pieces of $<1-0-0>$ silicon cut out from a plain, phosphorus doped, 4" wafer with a resistivity of 10-20 $\Omega \mathrm{cm}$ (Silicon Quest International). The functionalization steps were repeated on the large area TEOS sample in order to characterize the process. X-ray photoelectron spectroscopy (XPS) and contact angle measurements were performed to confirm the presence of the linkers and DNA (data not shown). The XPS studies were conducted on BL8.2 of the Stanford Synchrotron Radiation Laboratory (SSRL) at Stanford Linear Accelerator Center (SLAC) in Menlo Park, CA. The sulfur content of an oxide reference, a sample with linkers only and one with linkers and DNA were compared using XPS. The spectra recorded for the oxide reference indicated that no sulfur was present on the sample surface. In contrast, the appearance of sulfur $2 p_{3 / 2}$ and $2 p_{1 / 2}$ resonances in spectra of the linker and linker/DNA samples confirmed that the alkanethiol was bound to both substrates. An increase in the contact angle observed for the linker sample relative to the oxide reference was consistent with formation of a hydrophobic alkanethiol monolayer. 
Subsequent functionalization with the more hydrophilic DNA introduced a reduction in the contact angle relative to the linker sample. We also compared the nitrogen (via the magnitude of the $1 \mathrm{~s}$ resonance) content between reference, linker, and linker/DNA functionalized samples and recorded an increase in the nitrogen content after the DNA reaction, indicating the presence of DNA.

In order to demonstrate the DNA functionalization and verify the open nature of the nanopores, the ionic current across the devices was recorded. The silicon membrane holding the single pore was placed in a two-compartment cell containing an aqueous solution of $1 \mathrm{M} \mathrm{KCl}$ on both sides. Platinum electrodes were placed in each compartment and current-voltage (I-V) measurements were performed with a potentiostat (model 273A from Perkin Elmer) with a bias ranging from $1 \mathrm{~V}$ to 8V. Equation (1), where $\Phi$ represents the pore diameter, $\rho$ the ionic resistivity of the $\mathrm{KCl}$ solution, $\ell$ the pore length, and $\Delta V$ the transmembrane potential, was used to fit the current values obtained at $5 \mathrm{~V}$ for a series of devices with decreasing pore diameters. This formula defines the current measured, $I$, as the product of the transmembrane potential by the resistivity of the solution in the channel. ${ }^{17}$

$$
I=\Delta V\left(\frac{\Phi^{2} \pi}{4 \rho \ell}\right)
$$

A conductometer (model 712) from Metrohm was used to measure the conductivity of the $\mathrm{KCl}$ solution at $21^{\circ} \mathrm{C}$ and the value of the resistivity $\rho$ was found to be $\sim 11.7 \Omega \mathrm{cm}$. In the model, the 700 $\mathrm{nm}$ pore length was allowed to vary by $\pm 20 \mathrm{~nm}$, and the pore diameters by $10 \%$, to account for the resolution of the SEM measurement. We used pores with a starting diameter of $1000 \mathrm{~nm}$ to build the large apertures, and pores with starting diameters of $500 \mathrm{~nm}$ to build the smaller apertures. While equation (1), which describes a cylindrical pore, fits the pores with $1000 \mathrm{~nm}$ starting diameter quite well it was discovered that the pores with $500 \mathrm{~nm}$ starting diameter could not be fitted with this model without modifications to (1). While both pore types were perfectly cylindrical after initial drilling, SEM 
cross sections recorded after FIB cuts showed that after oxide deposition, the $1000 \mathrm{~nm}$ pores remained cylindrical but that the $500 \mathrm{~nm}$ pores were slightly conical, with a smaller top diameter. This can be explained by the fact that below a few hundred nanometers, the oxide deposition gradually transitions from a symmetrical deposition on the pore walls to a more localized deposition at the top opening, which results in an overall conical pore shape. The variation in geometry therefore has to be taken into account in order to fit the I-V data obtained for smaller pores, which can be done by replacing $\Phi^{2}$ in equation (1) by the product $\Phi \cdot \Phi^{*}$, $\Phi$ being the top pore diameter and $\Phi^{*}$ being the bottom pore diameter. ${ }^{17}$ The bottom pore diameter $\Phi^{*}$ was measured by SEM and was $430 \mathrm{~nm}$. Data for both pore types are shown in figure 5 below.

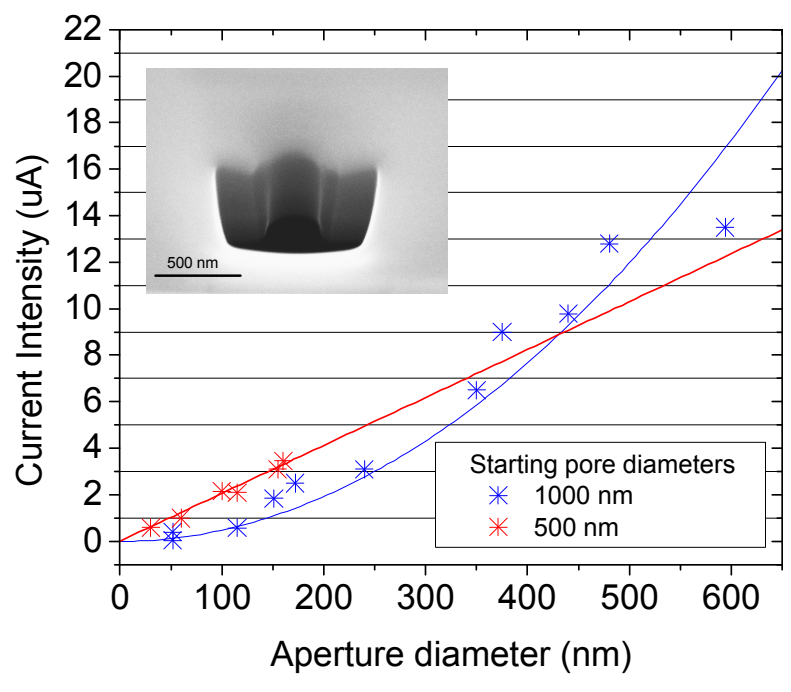

Figure 5. IV-measurements of single nanopores in $1 \mathrm{M} \mathrm{KCl}$ under a $5 \mathrm{~V}$ bias. The blue points represent data from the cylindrical pores and the red points show data from the conical pores. The red and blue lines are fits generated from the models discussed in the text. The SEM image in inset shows a cross section of a large cylindrical pore $(250 \mathrm{~nm})$ after a FIB cut. Cross sections of smaller pores are more difficult to analyze, due to material re-deposition during FIB milling. 
After DNA functionalization, IV-curves of the pores were re-measured under the same experimental conditions. The pores that were selected for functionalization were measured several times before any functionalization took place in order to ensure the accuracy of the ionic current values. Table 1 shows the experimental values obtained for a large range of pore diameters, as well as the current drop, $\Delta I$, calculated by inserting the linker-DNA length into equation (1) and calculating the difference between the before and after-functionalization current values. The distance between the silicon oxide surface and the end of the DNA probe is $7 \mathrm{~nm}$ for the 15-mer DNA strand anchored via the acrylamide group that we used.

\begin{tabular}{|c|c|c|c|}
\hline Sample & $\Delta \mathrm{I}_{\text {theory }}$ & $\Delta \mathrm{I}_{\text {experiment }}$ & $\varnothing(\mathrm{SEM})$ \\
\hline 1 (cylindrical) & $10 \%$ & $13 \%$ & $270 \mathrm{~nm}$ \\
\hline 2 (cylindrical) & $8 \%$ & $7 \%$ & $340 \mathrm{~nm}$ \\
\hline 3 (cylindrical) & $7 \%$ & $9 \%$ & $375 \mathrm{~nm}$ \\
\hline 4 (conical) & $20 \%$ & $20 \%$ & $70 \mathrm{~nm}$ \\
\hline 5 (conical) & $13 \%$ & $24 \%$ & $110 \mathrm{~nm}$ \\
\hline 6 (conical) & $9 \%$ & $11 \%$ & $155 \mathrm{~nm}$ \\
\hline
\end{tabular}

Table 1. Experimentally measured and theoretically calculated values of the ionic current drop induced by DNA functionalization of single nanopores with various size and shapes. The resolution of the $\mathrm{SEM}$ is $\sim 2-3 \mathrm{~nm}$ and the IV-setup is accurate to $1 \mathrm{xE}^{-8} \mathrm{~A}$. It is therefore reasonable to add an error margin of a few percent to the experimental data.

It is expected to see an increase of the current drop as the nanopores becomes smaller since the addition of DNA induces a larger relative reduction of the pore diameter on smaller features. The experimental results agree remarkably well with the theoretical calculations based on equation (1) for cylindrical pores and a modified version of equation (1) for conical ones. These results demonstrate that DNA probes were anchored on the silicon oxide ring surrounding the nanopores as a monolayer.

In conclusion, we fabricated single apertures on pre-patterned silicon platforms by Focused Ion Beam machining and we locally functionalized the pore entrances via the controlled growth of an oxide 
ring. Ionic current recorded through single nanopores at various stages of the fabrication process demonstrated that the apertures can be locally functionalized with DNA probes while remaining open. Focused Ion Beam nano-machining provides size tunability from microns to nanometers, which will allow biological objects ranging in size from cells down to viruses and DNA to be detected. In addition, the combination of ion-assisted deposition and silane chemistry provides local chemical functionality at the entrance of the nanopore, which will allow non-fouling coatings to be applied to the surface of the device for optimal stability and improved detection limits. Future applications for this functional platform include the selective detection of biological organisms and molecules by ionic current blockade measurements.

Acknowledgements. We thank the staff of the Microlaboratory of the University of California Berkeley, especially Jimmy Chang and Xiaofan Meng, for the assistance they provided with the equipment. We are also grateful to the staff of the National Center for Electron Microscopy at Lawrence Berkeley National Laboratory, especially Andrew Minor, for valuable help with the FIB. This work was performed under the auspices of the U.S. Department of Energy by University of California Lawrence Livermore National Laboratory under contract W-7405-Eng-48. It was funded by a Laboratory Directed Research and Development grant (LDRD-ER \# 03-ERD-013). 


\section{References}

(1) Henriquez, R. R.; Ito, T.; Sun, L.; Crooks, R. M. The Analyst 2004, 129, 478-482.

(2) Coulter, W. H. Means for Counting Particles Suspended in a Fluid 1953, US Patent No. 2656508.

(3) Chen, P.; Mitsui, T.; Farmer, D. B.; Golovchenko, J.; Gordon, R. G.; Branton. D. Nano Letters 2004, 4, 1333-1337.

(4) Saleh, O. A.; Sohn, L. L. Nano Letters 2003, 3, 37-38.

(5) Siwy, Z.; Apel, P.; Baur, D.; Dobrev, D. D.; Korchev, Y. E.; Neumann, R.; Spohr, R.; Trautmann, C.; Voss, K. Surface Science 2003, 532, 1061-1066.

(6) Mara, A.; Siwy, Z.; Trautmann, C.; Wan, J.; Kamme, F. Nano Letters 2004, 4, 497-501.

(7) Harrell, C. C.; Lee, S. B.; Martin, C. R. Anal. Chem. 2003, 75, 6861-6867.

(8) Chen, P.; Gu, J.; Brandin, E.; Kim, Y.; Wang, Q.; Branton, D. Nano Letters 2004, 4, $2293-$ 2298.

(9) Storm, A. J.; Storm, C.; Chen, J.; Zandbergen, H.; Joanny, J.; Dekker, C. Nano Letters 2005, 5, 1193-1197.

(10) Meller, A.; Nivon, L.; Brandin, E.; Golovchenko, J.; Branton, D. P. N. A. S. 2000, 97, 10791084.

(11) Bayley, H.; Cremer, P. S. Nature 2001, 413, 226-230.

(12) Howorka, S.; Cheley, S.; Bayley, H. Nature Biotech. 2001, 19, 636-639.

(13) Li, J.; Stein, D.; McMullan, C; Branton, D.; Aziz, M. J.; Golovchenko, J. A. Nature 2001, 412, 166-169.

(14) Harrell, C. C.; Kohli, P.; Siwy, Z.; Martin, C. R. J. Am Chem. Soc. 2004, 126, 15646-15647.

(15) Young, R. J.; Puretz, J. J. Vac. Sci. Technol. B 1995, 13, 2576-2579.

(16) Edinger, K.; Melngailis, J.; Orloff, J. J. Vac. Sci. Technol. B 1998, 16, 3311-3314.

(17) Siwy, Z.; Fuliński, A. Am. J. Phys. 2004, 72, 567-574. 
J. Nilsson et al.

NanoLetters

Table of content

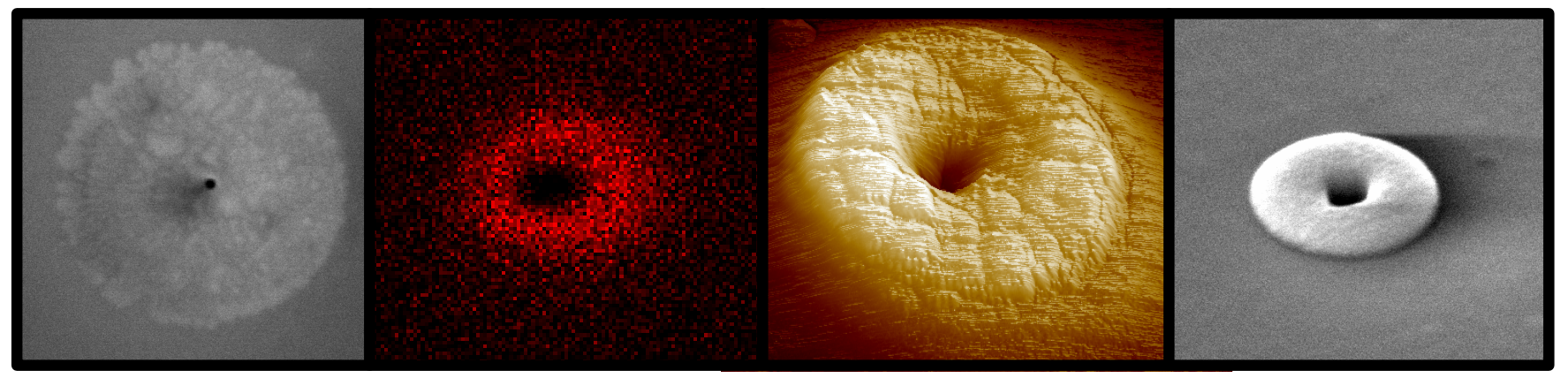

